\section{Effects of Temperature during Irradiation on the Production of 2-Alkylcyclobutanones in Beef}

\author{
Hirotaka Obana, ${ }^{*, a}$ Masakazu Furuta, ${ }^{b}$ \\ and Yukio Tanaka ${ }^{a}$
}

${ }^{a}$ Osaka Prefectural Institute of Public Health, 1-3-69 Nakamichi, Higashinari, Osaka 537-0025, Japan and ${ }^{b}$ Osaka Prefecture University Graduate School of Science, 1-2 Gakuen-cho, Naka, Sakai, Osaka 599-8570, Japan

(Received September 5, 2006; Accepted December 5, 2006; Published online January 10, 2007)

Beef patties were irradiated with $\gamma$-rays at three temperatures, room temperature, chilled, and frozen. 2-Alkylcyclobutanones in irradiated beef patties were then analyzed to confirm the effects of the three temperatures during irradiation on their production. The production of 2-dodecylcyclobutanone (2DCB) and 2-tetradecylcyclobutanone (2-TCB) were decreased in the samples cooled with ice and dry ice compared with those at room temperature. 2-DCB was dominant in the samples irradiated at room temperature, which reflected the composition of the precursor of the fatty acid in the samples, whereas they became lower than 2-TCB in the samples irradiated with cooling. The data indicate that the temperature during irradiation greatly affects the radiolytic production of 2-alkylcyclobutanones, particularly 2DCB, as well as precursor concentrations.

Key words — 2-alkylcyclobutanone, irradiation, temperature, beef, cooling

\section{INTRODUCTION}

The ionizing radiation of food is used to retain its safety and quality by controlling microorganisms and extending shelf life. ${ }^{1,2)}$ The irradiation of food has been permitted at regulated doses in many countries. $^{3,4)}$ Confirming the irradiation history of food would facilitate consumers' choices and acceptance of irradiated food, and may help in the enforcement of labeling regulations.

\footnotetext{
*To whom correspondence should be addressed: Osaka Prefectural Institute of Public Health, 1-3-69 Nakamichi, Higashinari, Osaka 537-0025, Japan. Tel.: +81-6-6972-1321; Fax: +81-6-6972-2393; E-mail: obana@iph.pref.osaka.jp
}

Numerous methods have been developed to detect irradiated foodstuffs. ${ }^{5}$ ) European countries have legitimately adopted the analysis of 2alkylcyclobutanones as EN 1785 to detect irradiated food containing fat, such as chicken, pork, and eggs. ${ }^{6)}$ 2-Alkylcyclobutanones are considered to be unique radiolytic products of fatty foods with irradiation. 2-Dodecylcyclobutanone (2-DCB) from palmitic acid and 2-tetradecylcyclobutanone (2-TCB) from stearic acid have been recommended as markers for the irradiation of lipid-containing food. $^{7-10)}$

We have developed a method to detect irradiated food by analyzing 2-DCB and 2-TCB and found that the production of 2-DCB was less than that of 2-TCB, whereas the concentrations of palmitic acid were greater than those of stearic acids in beef and pork samples irradiated under frozen conditions. ${ }^{11)}$ This result contradicts with the commonly accepted belief that the amounts of radiolytically produced 2alkylcyclobutanones constantly reflect the composition of precursors in the samples. $\left.{ }^{9}, 12,13\right)$ One possible contributor affecting the radiolytic production of 2-alkylcyclobutanones might be the temperature during irradiation. Ndiaye et al. proposed that lower temperature would decrease $\mathrm{OH}$ radicals induced by irradiation, which lead to the production of 2alkylcyclobutanones. ${ }^{12)}$ The objective of this study was to confirm the effects of temperature during irradiation of beef patties and precursor compounds on the radiolytic production of 2-DCB and 2-TCB.

\section{MATERIALS AND METHODS}

Reagents - 2-DCB and 2-TCB were obtained from Fluka (Steinheim, Switzerland), and other reagents were obtained from Wako Pure Chemical (Osaka, Japan).

Food Commodities _- Ground beef was purchased at a local market in Osaka, Japan. Samples of approximately $500 \mathrm{~g}$ were chopped in a conventional food processor to obtain a homogenous fat distribution in the ground beef. Patties ( $40 \mathrm{~g}$ each) with a diameter of $95 \mathrm{~mm}$ and thickness of $6 \mathrm{~mm}$ were prepared from the ground beef as described previously. $^{14)}$

Irradiation — Samples were irradiated with $\gamma$ rays from a ${ }^{60} \mathrm{Co}$ source $(15 \mathrm{kGy} / \mathrm{h})$ in the irradiation pool at the Frontier Science Innovation Center, Osaka Prefecture University, as described previously. ${ }^{11)}$ During irradiation with $5.3 \mathrm{kGy}$, the sam- 
ples were subjected to three temperature conditions: room temperature of about $20^{\circ} \mathrm{C}$; ice-cooled to about $0^{\circ} \mathrm{C}$; and dry ice-cooled, to $-78^{\circ} \mathrm{C}$. The samples were placed in a container packed with crushed ice or powdered dry ice to cool.

An aliquot of $3 \mathrm{ml}$ of either palmitic acid, stearic acid, methyl palmitate, or methyl stearate $1 \mathrm{mmol} / \mathrm{l}$ in hexane was also irradiated together with the patties. An aliquot of $3 \mathrm{ml}$ of either tripalmitin or tristearin $0.333 \mathrm{mmol} / \mathrm{l}$ in $n$-hexane was also irradiated. Both tripalmitin and tristearin are esterified compounds of one glycerol molecule with three palmitic acid and three stearic acid molecules, respectively. The triglycerides contain 3-fold the amount of free fatty acid; thus the irradiated concentration would correspond to $1 \mathrm{mmol} / \mathrm{l}$ of fatty acid on a precursor basis.

The irradiation dose was determined with Radiachromic dye film as described previously. ${ }^{11)}$ The irradiated meat was stored at $-20^{\circ} \mathrm{C}$ until the analysis of 2-alkylcyclobutanones.

Sample Preparation for Analysis — Sample preparation for the analysis of 2-DCB and 2-TCB was performed as previously described. ${ }^{11)}$ Briefly, $10 \mathrm{~g}$ each of sample and diatomaceous earth were thoroughly mixed, and the mixture was extracted with ethyl acetate at $100^{\circ} \mathrm{C}$ using an accelerated solvent extraction system. An aliquot of $10 \mathrm{ml}$ of extract in $50 \mathrm{ml}$ of extract and $10 \mathrm{ml}$ of acetonitrile was mixed and kept at $-20^{\circ} \mathrm{C}$ to precipitate the fat, which was removed by immediate filtration. The fat precipitation and filtration were repeated to remove the remaining fat in the first filtrate. The second filtrate was evaporated, dissolved in $n$-hexane, and added to a silica gel cartridge (Mega Bond Elut SI, $1 \mathrm{~g}$, Varian, CA, U.S.A.). $n$ Hexane $10 \mathrm{ml}$ was eluted and discarded. Then, $10 \mathrm{ml}$ of $2 \%$ diethyl ether in $n$-hexane was further eluted and collected. After the addition of 2cyclohexylcyclohexanone, the eluate was concentrated to $0.1 \mu \mathrm{g} / \mathrm{ml}$. Twenty-five milliliters of the remaining extract was evaporated to almost dryness in preweighed round-bottomed flasks, and the fat residue was weighed after standing overnight for the calculation of the fat basis concentration.

2-DCB and 2-TCB were determined with gas chromatography-mass spectrometry (GC/MS), the details of which were described elsewhere. ${ }^{11,14)}$

Fatty Acids — Fatty acids in the weighed fat were determined using an established method. ${ }^{15)}$ Methylated fatty acids were determined with GC/MS monitored at $m / z 87$.
Statistics - 2-DCB and 2-TCB in the chilled or frozen samples and those in the uncooled samples were statistically compared using Student's $t$-test with Microsoft Excel 2004 for Macintosh.

\section{RESULTS AND DISCUSSION}

The 2-alkylcyclobutanone concentrations in the irradiated patties at three temperatures are illustrated in Fig. 1, as expressed on the fat basis. The 2-DCB concentration in the chilled samples was significantly $(60 \%)$ lower than that in the samples without cooling, and about a further $10 \%$ lower in the samples frozen with dry ice during irradiation. The 2-TCB concentration in the samples chilled during irradiation was also significantly lower by about $30 \%$ than that in the samples without cooling, and about a further $10 \%$ lower in the samples frozen with dry ice during irradiation. The ratio of 2-DCB to 2-TCB without cooling was about 1.62, which was higher than the 0.88 and 0.68 of the samples irradiated with cooling. The decreased ratios indicate that the 2-DCB concentrations were reduced more than the 2-TCB concentrations in the samples cooled during irradiation. GC/MS chromatograms of the irradiate samples are illustrated in Fig. 2. The chromatograms indicate that the two compounds were clearly distinguishable from other coexisting peaks, and the two peaks decreased as the temperatures of samples during irradiation decreased.

2-DCB and 2-TCB were produced from palmitic acid and stearic acid, respectively, in the samples. Their production was considered to reflect

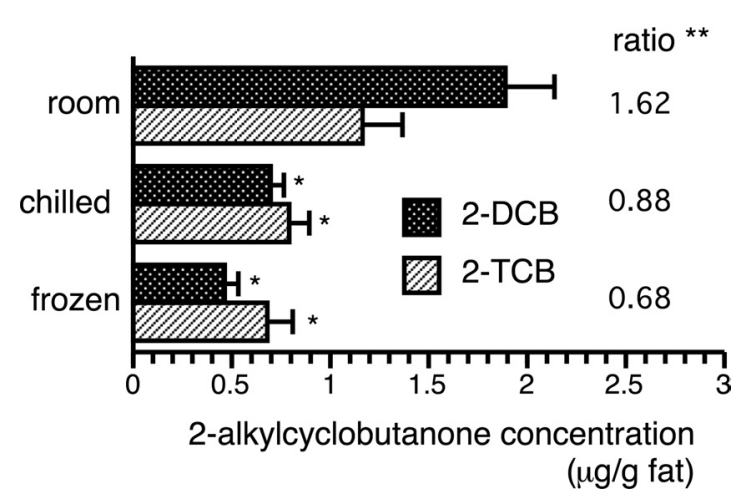

Fig. 1. Effects of Irradiation Temperature on the Production of 2-DCB and 2-TCB in Beef Patties

2-DCB and 2-TCB concentrations are the average of five measurements expressed as the fat basis. *Significantly different from room temperature, Student's $t$-test. ${ }^{* *}$ Ratio of 2-DCB to 2-TCB. 
the respective precursor fatty acid levels in the samples. ${ }^{10,13)}$ The average concentrations of palmitate and stearate in the samples were $28.8 \pm 2.3$ (average \pm standard deviation, $n=15)$ and $15.6 \pm$ $1.0 \mathrm{mg} / \mathrm{g}$ on a weight basis, respectively (data not shown). The ratio of palmitic acids to stearic acids ranged from 1.82 to 1.87 , which was similar to the ratio of 2-DCB to 2-TCB, the respective radiolytic products, with irradiation at room temperature, as shown in Fig. 1.

The 2-alkylcyclobutanone concentrations are expressed based on the respective precursor compound basis to clarify the relation of the product and the precursor with irradiation at the three temperature conditions (Fig. 3). If 2-DCB and 2-TCB could be produced in the same proportion from the respec-

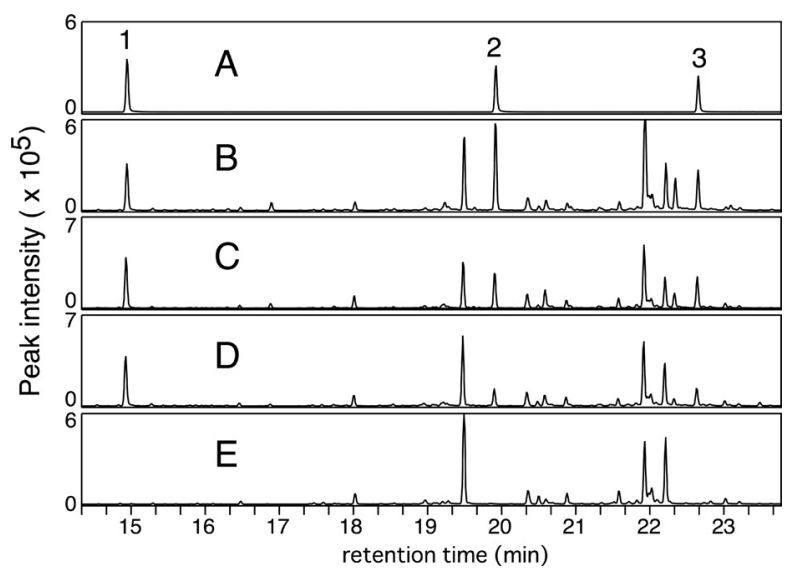

Fig. 2. Typical Chromatograms of 2-Alkylcyclobutanones in Irradiated Beef Patties at Three Temperatures Monitored at $m / z, 98$

(A) 2-DCB and 2-TCB $100 \mathrm{ng} / \mathrm{ml}$, (B) irradiated at room temperature, (C) irradiated with ice, (D) irradiated frozen with dry ice, (E) nonirradiated raw beef. Peak labels: 1,2-cyclohexylcyclohexanone as internal standard; 2,2-DCB; 3,2-TCB. tive precursors, the ratio of the two product concentrations would become one. Figure 3 indicates that the production of the two compounds was at similar levels when the samples were not cooled during irradiation. The production of the two compounds decreased as the irradiation temperature decreased, and the ratio of the two compounds decreased to less than one-half in the frozen samples. The data indicate that the radiolytic production of both compounds at room temperature would reflect the precursor levels as expected; however, their production was inhibited when irradiated with cooling, particularly that of 2-DCB.

The inhibitory effects of temperature on 2-DCB and 2-TCB production were studied under simple conditions. A precursor compound in $n$-hexane solution was irradiated under the same conditions as described above (Table 1). Some of the produc-

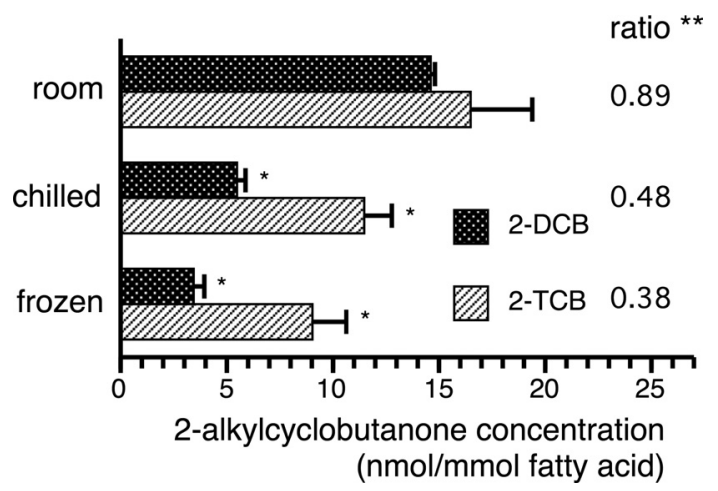

Fig. 3. Effects of Irradiation Temperature on the Production of 2-DCB and 2-TCB in Beef Patties

2-DCB and 2-TCB concentrations are the average of five experiments expressed as the respective precursor basis. ${ }^{*}$ Significantly different from room temperature, Student's $t$-test. ${ }^{* *}$ Ratio of 2-DCB to 2-TCB.

Table 1. Radiolytic Production of 2-DCB and 2-TCB from Respective Precursors

\begin{tabular}{|c|c|c|c|c|c|c|}
\hline Precursor $^{a)}$ & Temperature & $\begin{array}{c}\text { 2-DCB average } \\
(\mathrm{nmol} / \mathrm{l})\end{array}$ & $\overline{\text { S.D. }^{c)}}$ & $\begin{array}{c}\text { 2-TCB average } \\
(\mathrm{nmol} / \mathrm{l})\end{array}$ & 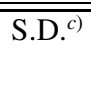 & 2-DCB/2TCB \\
\hline \multirow[t]{3}{*}{ Free acid } & Room & 134 & 10 & 167 & 5 & 0.80 \\
\hline & Chilled & 102 & 14 & 141 & 47 & 0.72 \\
\hline & Frozen & - d) $^{d}$ & & -d) $^{d}$ & & \\
\hline \multirow[t]{3}{*}{ Methyl ester } & Room & 73 & 6 & 110 & 7 & 0.66 \\
\hline & Chilled & 77 & 9 & 95 & 1 & 0.81 \\
\hline & Frozen & 14 & 9 & 18 & 17 & 0.79 \\
\hline \multirow[t]{3}{*}{ Triglyceride ester } & Room & 92 & 13 & 132 & 4 & 0.70 \\
\hline & Chilled & 60 & 18 & -d) $^{d}$ & & \\
\hline & Frozen & - d) $^{-1}$ & & -d) $^{d}$ & & \\
\hline
\end{tabular}

a) Precursor compounds were irradiated with $5.3 \mathrm{kGy} \gamma$-rays. Palmitic acid was irradiated for 2-DCB and stearic acid was irradiated for 2-TCB. $b$ ) Average of three measurements. $c$ ) Standard deviation. $d$ ) Compound was precipitated. 
tion levels were unfortunately not available because the precursors precipitated under the cooled conditions, and thus their original concentrations could not be reached during irradiation, and the product levels were markedly low. The radiolytic production levels of the two compounds, for which the corresponding precursors were set at $1 \mathrm{mmol} / \mathrm{l}$, varied within the range of 2-fold with irradiation at room temperature, and thus the values were comparable although the precursors were different. The results indicated that 2-DCB production was slightly lower than that of 2-TCB, which resembled the ratio in beef patties without cooling as shown in Fig. 3. The production ratio of 2-DCB to 2-TCB did not decrease with ice-cooled irradiation with free acid and methyl ester. Even with dry ice-cooled methyl ester, the production ratio did not change, although the low temperature markedly inhibited the production of both compounds in the patties. Ndiaye et al. proposed that irradiation produced fewer $\mathrm{OH}$ radicals in frozen samples than in uncooled ones, ${ }^{12)}$ and thus the production of 2-alkylcyclobutanones decreased. The fat in the beef patties was in a solid state even at chilled temperature, which would result in a decrease in radiolytic products. The results lead to the conclusion that the decrease in 2-DCB under cooled irradiation might be related to the sample matrix, because such a decrease was not observed in the simple solutions of precursor; however, the mechanism is not clear.

Gadgil et al. indicated that the levels of 2alkylcyclobutanones detected could predict the irradiation doses, because the concentration of 2-DCB increased linearly with the dose, and there was no significant difference in the 2-DCB concentrations among samples with different fat levels. ${ }^{16)}$ The data in this study show that another factor, the temperature during irradiation, should be take into account when predicting the irradiation dose based on the 2alkylcyclobutanone concentration produced. The irradiation of meat is usually performed under chilled or frozen conditions since it is irradiated in the distribution system after packing. The results of this study contribute to bridging the gap between 2alkylcyclobutanone residues and the their precursor compositions in irradiated samples.

Acknowledgements This work was supported in part by a Grant-in-Aid for Scientific Research from the Ministry of Education, Culture, Sports, Science and Technology of Japan.

\section{REFERENCES}

1) Diehl, J. F. (2001) Achievements in food irradiation during the 20th century. In Irradiation for Food Safety and Quality (Loaharanu, P. and Thomas, P., Eds.), Technomic Publishing, Lancaster, U.K., pp. $1-16$.

2) Molins, R. A. (Ed.) (2001) Food Irradiation: Principles and Applications, Wiley-Inter Science, New York, pp. 23-339.

3) Pauli, G. H. and Tarantino, L. M. (1995) FDA regulatory aspects of food irradiation. J. Food Prot., 58, 209-212.

4) Vounakis, H. (2001) Food irradiation harmonization in the European Union. In Irradiation for Food Safety and Quality (Loaharanu, P. and Thomas, P., Eds.), Technomic Publishing, Lancaster, U.K., pp. 26-31.

5) McMurray, C. H., Stewart, E. M., Gray, R. and Pearce, J. (Eds.) (1996) Detection Methods for Irradiated Foods: Current Status. Royal Society of Chemistry, Cambridge, U.K., pp. 3-414.

6) European Committee for Standardization, (2003) Foodstuffs-Detection of Irradiated Food Containing Fat: Gas Chromatographic/Mass Spectrometric Analysis of 2-Alkylcyclobutanones EN1785. European Committee for Standardization, Brussels.

7) Stevenson, M. H., Crone, A. V. J. and Hamilton, J. T. G. (1990) Irradiation detection. Nature, 344, 202203.

8) Boyd, D. R., Crone, A. V. J., Hamilton, J. T. G., Hand, M. V., Stevenson, M. H. and Stevenson, P. J. (1991) Synthesis, characterization and potential use of 2-dodecylcyclobutanone as marker for irradiated chicken. J. Agric. Food Chem., 39, 789-792.

9) Crone, A. V. J., Hamilton, J. T. G. and Stevenson, M. H. (1992) Effect of storage and cooking on the dose response of 2-dodecylcyclobutane, a potential marker for irradiated chicken. J. Sci. Food Agric., 58, 249-252.

10) Crone, A. V. J., Hand, M. V., Hamilton, J. T. G., Sharma, N. D., Boyd, D. R. and Stevenson, M. H. (1993) Synthesis, characterization and use of 2-tetradecylcyclobutanone together with other cyclobutanones as markers for irradiated liquid whole egg. J. Sci. Food Agric., 62, 361-367.

11) Obana, H., Furuta, M. and Tanaka, Y. (2005) Analysis of 2-alkylcyclobutanones with accelerated solvent extraction to detect irradiated meat and fish. $J$. Agric. Food Chem., 53, 6603-6608.

12) Ndiaye, B., Jametb, G., Miesch, M., Hasselmann, C. and Marchioni, E. (1999) 2-Alkylcyclobutanones as markers for irradiated foodstuffs II. The CEN (Euro- 
pean Committee for Standardization) method: field of application and limit of utilization. Radiat. Phys. Chem., 55, 437-445.

13) Stevenson, M. H. (1994) Identification of irradiated foods. Food Technol., 48, 141-144.

14) Obana, H., Furuta, M. and Tanaka, Y. (2006) Detection of 2-alkylcyclobutanones in irradiated meat, poultry and egg after cooking. J. Health. Sci., 52, 375-382.
15) Pharmaceutical Society of Japan (Ed.) (2000) Methods of Analysis in Health Science 2000, Kanehara \& Co., Tokyo, pp. 194-196.

16) Gadgil, P., Smith, J. S., Hachmeister, K. A. and Kropf, D. H. (2005) Evaluation of 2dodecylcyclobutanone as an irradiation dose indicator in fresh irradiated ground beef. J. Agric. Food Chem., 53, 1890-1893. 